\title{
CONCENTRATION OF CD8+ LYMPHOCYTES ON EWOD PLATFORM FOR MONITORING ORGAN TRANSPLANT REJECTION
}

Gaurav J. Shah ${ }^{1}$, Jeffrey L. Veale, Yael Korin ${ }^{3}$, Elaine F. Reed ${ }^{3}$, H. Albin Gritsch', and Chang-Jin “CJ” Kim ${ }^{1}$

${ }^{1}$ Mechancial and Aerospace Engineering Department, Henry Samueli School of Engineering and Applied Science

${ }^{2}$ Department of Urology, ${ }^{3}$ Immunogenetics Center, David Geffen School of Medicine

University of California, Los Angeles (UCLA), Los Angeles, CA 90095

\begin{abstract}
As important developments in the effort to create a low-power portable lab-on-a-chip system, we demonstrate the concentration of human CD8+ T-lymphocytes on an electrowetting-on-dielectric (EWOD) platform using antibody-conjugated magnetic beads (MB-Ab). This is an important step of the protocol used to monitor the risk of rejection in organ transplant patients.
\end{abstract}

\section{INTRODUCTION}

The ability to concentrate and separate specific cells is critical for cell-based biological assays and medical diagnostics. For instance, CD8+ cytotoxic T-lymphocytes play a critical role in transplantrejection by infiltrating the transplanted organs and releasing proteins that attract additional lymphocytes to destroy the transplant cells. If CD8+ lymphocytes could be isolated from other peripheral blood components and then lysed, the concentration of these cells and their associated proteins could be measured to diagnose rejection non-invasively $[1,2]$. While this protocol is well-established at the UCLA Immunogenetics Center, the transplanted patients have to visit the center for the tests. A portable device performing the lab test would not only obviate the post-transplantation visits but also facilitate early diagnosis and timely treatment. With its simplicity and low power-consumption, electrowetting-on-dielectric (EWOD)-based digital microfluidics is promising for such a point-of-care device.

In the quest to realize the entire lab protocol on a portable system, we report two main steps realized on the EWOD platform. First, we developed and quantitatively assessed binding of CD8+ cells to MB-Abs. Second, we developed the ability to separate CD8+ lymphocytes from a mixture of CD8+ and CD8lymphocytes. While recent studies have shown concentration of MBs on EWOD platform is feasible in principle using simple water containing only the beads [3-5], this is the first report of success in advancing the concept to living cells.

\section{MATERIALS AND METHODS}

\section{Cells Sample Preparation}

Lymphocytes were isolated from whole blood using standard hematological procedures. Peripheral blood mononuclear cells were separated over a Ficoll-Hypaque gradient. Lymphocytes were obtained after macrophage depletion by adherence to a plastic flask. CD8+ and CD8- lymphocytes were separated using antiCD8 MB-Ab (Dynabeads CD8 Positive Isolation Kit from Invitrogen, Inc., CA) before being detached from the MB's. Before EWOD experiments, the cells were spun down and resuspended in serum-free buffer. For visualization, the CD8+ cells were stained with fluorescent dye (CFSE).

\section{EWOD Device Fabrication}

Typical UCLA EWOD fabrication processes [3] were used to prepare the device (Fig. 1). EWOD electrodes were defined on an Indium-Tin Oxide (ITO) (1400 ̊) layer over a $700 \mu$ m-thick glass (“EWOD”) substrate (TechGophers Inc.). Cr/Au ( 100/1000 ̊́) was deposited and patterned to define the contact pads and electrode labels for easier visualization. Next, a $\mathrm{Si}_{3} \mathrm{~N}_{4}$ layer $(\sim 1$ $\mu \mathrm{m})$ was deposited using PECVD, and patterned to define the dielectric layer. A Cytop ${ }^{\circledR}$ (Asahi Inc.) layer $(\sim 1 \mu \mathrm{m})$ was spincoated on top, and annealed at $200{ }^{\circ} \mathrm{C}$ to make the surface hydrophobic. $1.1 \mathrm{~mm}$-thick glass substrates coated with ITO (1400 $\AA$ ) (Delta Technologies Inc.) were used to fabricate the "Reference" substrate. A thinner PECVD $\mathrm{Si}_{3} \mathrm{~N}_{4}$ layer $(\sim 1000 \AA ̊)$ was deposited and patterned on it to expose the ITO for electrical ground connection, followed by Cytop ${ }^{\circledR}$ spin-coating and annealing ( 1000 ̊̊). A double-sided tape ( 100 $\mu \mathrm{m}$ thick, 3M Inc.) was used as the spacer between the substrates.

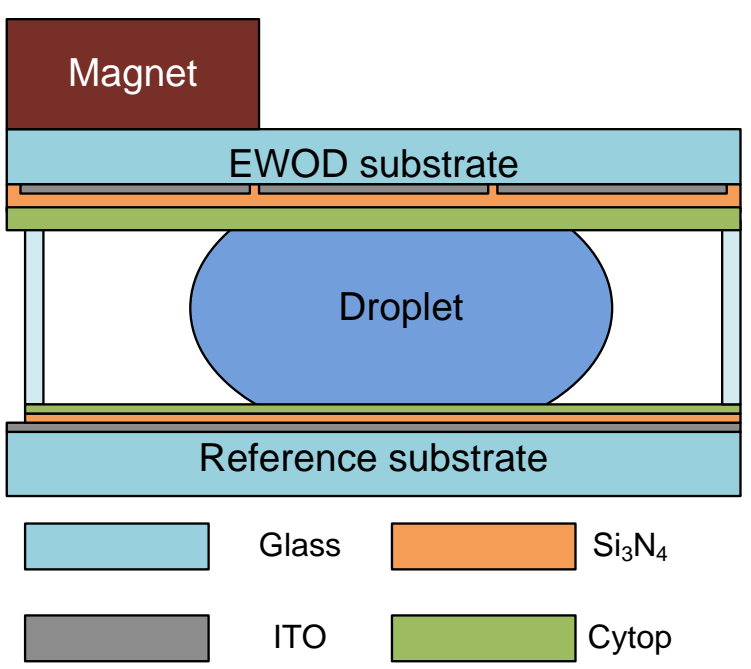

Figure 1: Cross section of the EWOD device (not precisely to scale). EWOD actuation electrodes were patterned on the ITO layer of the "EWOD" substrate. Contact pads were formed with $\mathrm{Cr} / \mathrm{Au}$ (not shown). Electrodes were coated with silicon nitride as dielectric and cytop as hydrophobic coating. Thinner silicon nitride and cytop layers were deposited over the ITO-coated "Reference" substrate, connected to ground.

\section{Device Actuation and Image Capture}

Droplet actuation was achieved by application of voltage ( 70 Vac $1 \mathrm{kHz}$ ) to EWOD electrodes. Electronic control for the actuation sequence was controlled using LabVIEW (National Instruments Inc.) with the help of a digital I/O device (DAQPad 6507, National Instruments). Magnetic force was provided using a cylindrical permanent magnet ( $\mathrm{NdFeB}, 1 / 2$ " dia. $1 / 2$ " thick) placed on top of the EWOD substrate (Fig. 1).

The device was mounted on an inverted fluorescence microscope (Nikon TE-2000U) for visualization. A video camera (Panasonic KR-222) was used to capture the droplet actuation movies, while still optical and fluorescence images were taken using a cooled CCD camera (Photometrics Coolsnap EZ).

\section{RESULTS AND DISCUSSION}

Flow cytometry Experiments

Before doing experiments on EWOD, the assay was verified using the conventional laboratory techniques. 

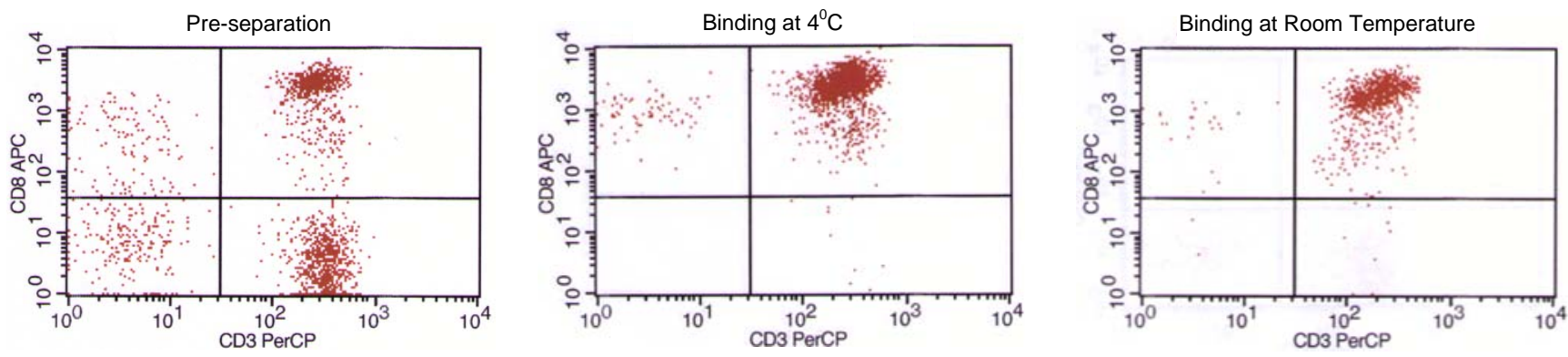

Figure 2: Test of the protocol to bind CD8+ cells to MBs under EWOD conditions. Flow cytometry data obtained for lymphocyte distribution before and after magnetic separation. Upper right corner indicates CD8+ T-lymphocytes. (Left) Before separation. (Middle) after magnetic separation for MB-cell binding done at $4^{0} \mathrm{C}$ as per protocol, (Right) after magnetic separation for MB-cell binding at RT in conditions similar to those during EWOD experiments. Collection efficiency is high (>95\%) in both cases.

Flow-cytometry measurements were performed to determine the isolation efficiency for cell separation according to protocol. To ensure that MB-cell binding will also occur on an EWOD device, cell separation was also performed for binding conditions similar to EWOD (e.g. room temperature instead of the prescribed $2-8^{\circ} \mathrm{C}$ ). Fig. 2 shows that CD8+ isolation efficiency was similarly high (>95\%) under both conditions (Fig. 2 middle, right).

\section{Cell-MB Binding on EWOD}

The steps to evaluate CD8+ cells binding with MBs on the EWOD device are schematically described in Fig. 3 and demonstrated in Fig. 4. A droplet containing MB-Ab $\left(\sim 10^{7} / \mathrm{ml}\right)$ and another containing fluorescently stained CD8+ cells $\left(\sim 10^{5} / \mathrm{ml}\right)$ were merged using EWOD (Figs. 3(a, b), 4(a, b)). The combined droplet was moved repeatedly over a circular path of electrodes to allow MB-cell mixing. After about 8-10 minutes (Fig. 3(c, d), 4(c, d)), an
NdFeB magnet was introduced (Fig. 3(e), 4(e)), attracting most MBs to the left-end of the droplet. (Earlier reports of MB separation on EWOD used meniscus-assistance [3], or performed separation immediately after sample introduction [4]. Although they are valid, a proprietary technique by Core Microolutions, Inc. (Los Angeles, CA) used here eliminates the need for either of these.) The droplet is subsequently cut to form the collected droplet (left) and the depleted droplet (right) (Fig. 5(a, b)).

By manually counting the fluorescent cells in these droplets, the cell-collection efficiency is conservatively estimated to be $>92 \%$ (Fig. 5(c-f)). (Some cells in the collected droplet may be hidden behind MBs.). This high efficiency is attributed to the high interaction between MBs and cells confined inside a circulating droplet, as compared to a flow-through channel-microfluidics system.

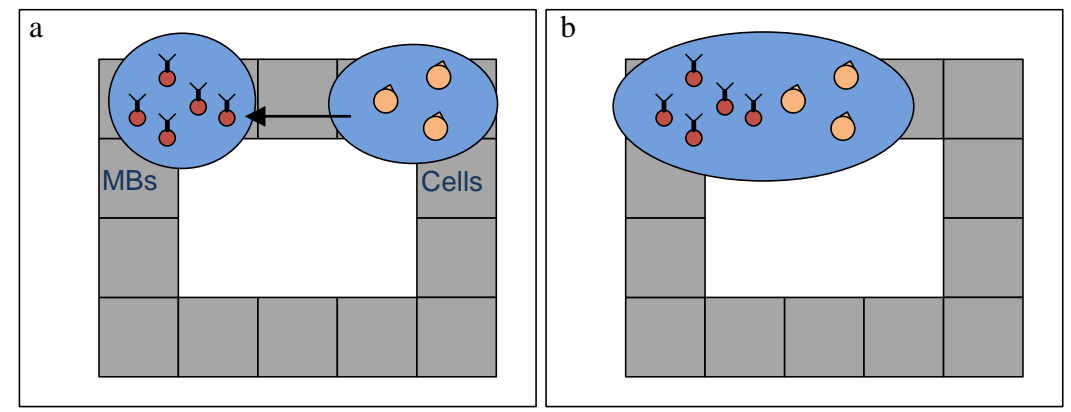

Figure 3: Schematic description of binding cells to MBs and concentration of the cells on EWOD platform. $(a, b)$ A droplet containing MBs and another containing cells are merged. $(c, d)$ Merged droplet is repeatedly moved around using EWOD along a circular path to mix the MBs and cells. (e) Magnet pulls MBs to the left end, along with the bound cells.
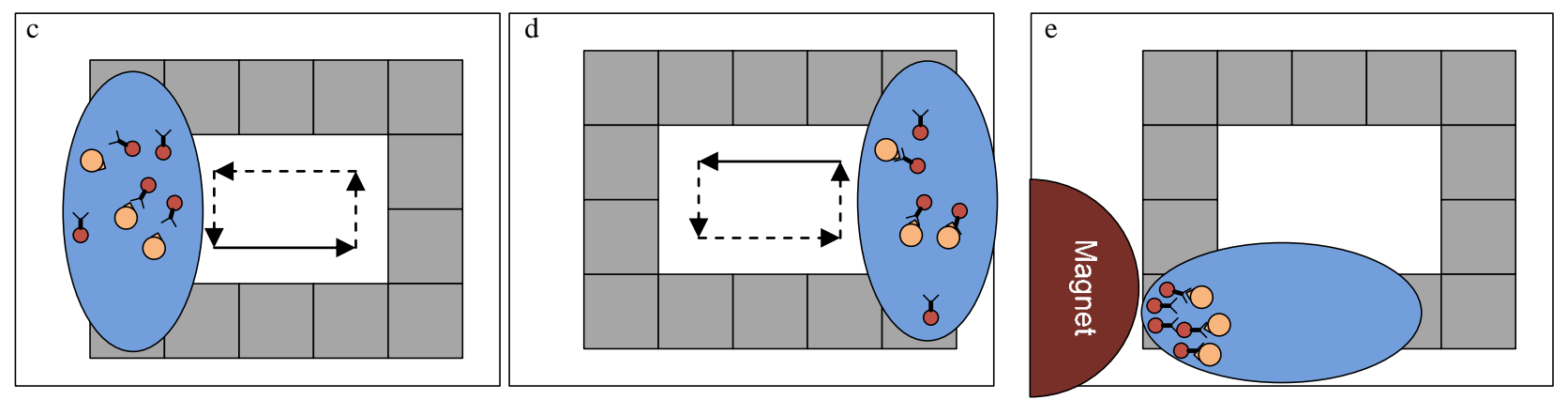

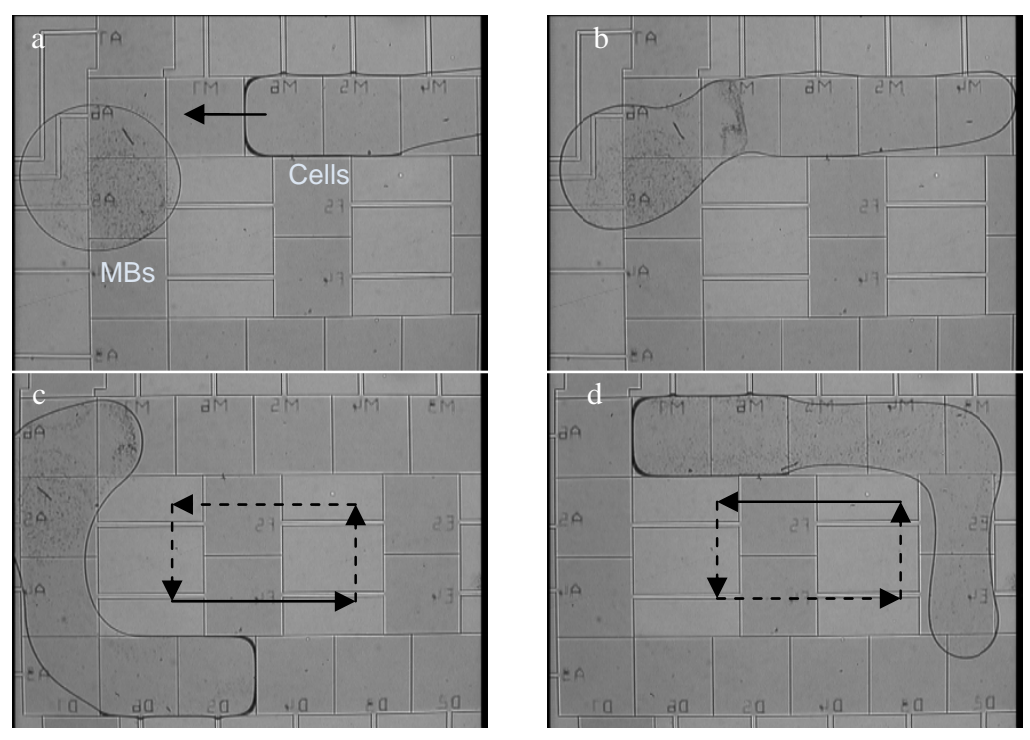

Figure 4: Image-sequence for $C D 8+T$ lymphocytes binding to MBs on EWOD. $(a, b)$ Droplets containing MBs and cells are merged. $(c, d)$ Droplet is repeatedly moved around using EWOD (for 8-10 minutes here), along a circular path. (e) Magnet pulls MBs to the left end, along with the bound CD8+ T-lymphocytes.

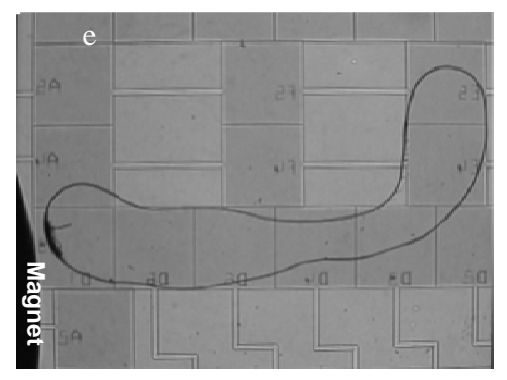

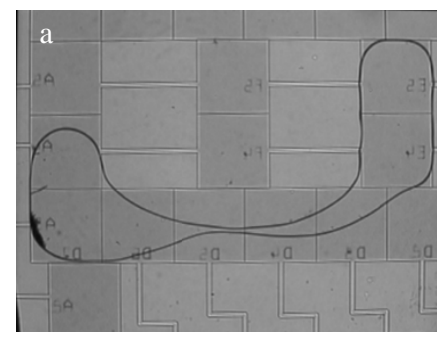

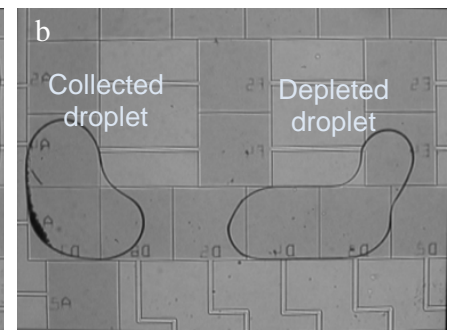

Figure 5: Assessment of binding efficiency. $(a, b)$ Droplet is split after binding. Most CD8+ cells are bound to MBs and collected in the left droplet, as seen in the bright-field and fluorescence images. (c, f) Initial cell sample before merging with MB-containing droplet: 88 stained cells counted.

After binding, $(d, g)$ Collected droplet: $~ 81$ stained cells counted (Some may be hidden behind MBs.) $(e, h)$ Depleted droplet: < 2 stained cells counted.
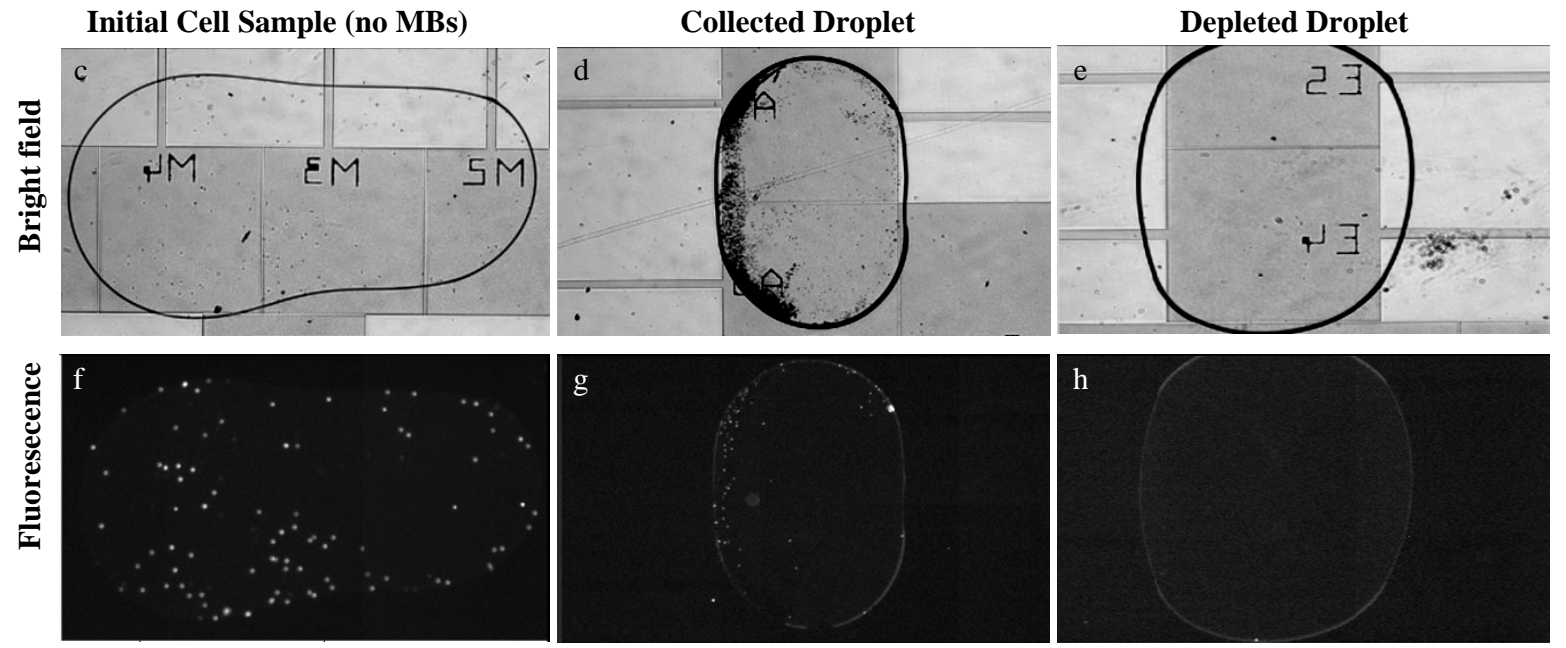

\section{Separation of CD8+ and CD8- lymphocytes}

Fig. 5 shows the ability to separate CD8+ from CD8- cells. The initial sample contains fluorescently stained CD8+ cells preconjugated with MBs, mixed with unlabeled CD8- cells ( $10^{5} / \mathrm{ml}$ total) (Fig. 6(a), 7(a,e)). On introduction of a magnet, the MB-CD8+cells move to the left meniscus (Fig. 6(b), 7(b, f)). Fluorescence can be observed where MBs are. The droplet is then cut using EWOD (Fig. 6(c, d), 7(c, d)), so that most MB-bound CD8+ cells are now collected in the left droplet.

Since they are not actively removed, some CD8- cells may also be collected. However, by adding wash buffer and repeating similar steps as above (Fig. 6(d,e)), CD8- cells can be eventually depleted, leaving mostly CD8+ cells in the final collected droplet (Fig. 6(f)). 

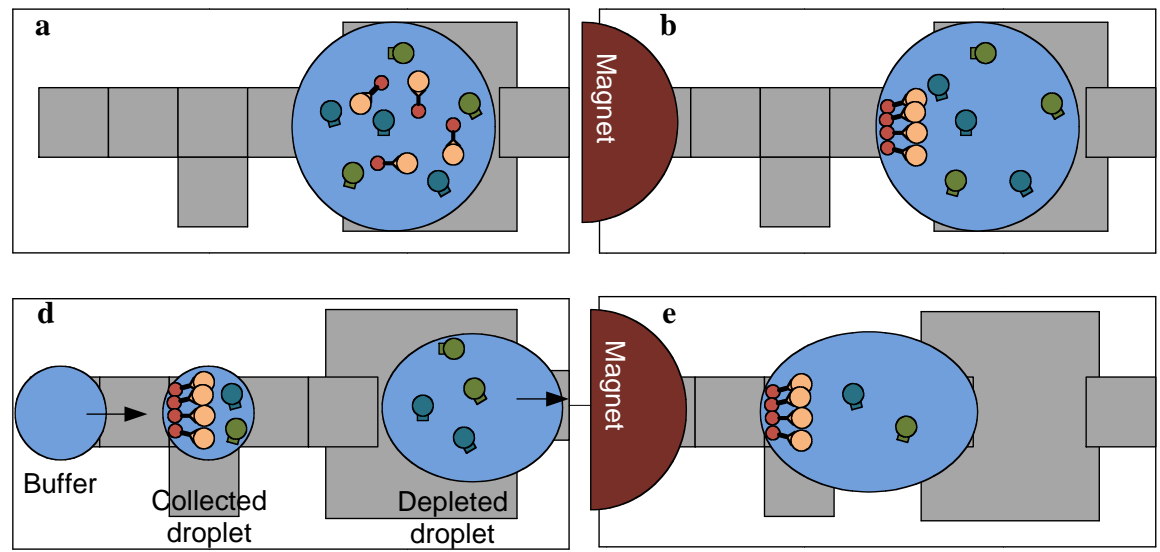
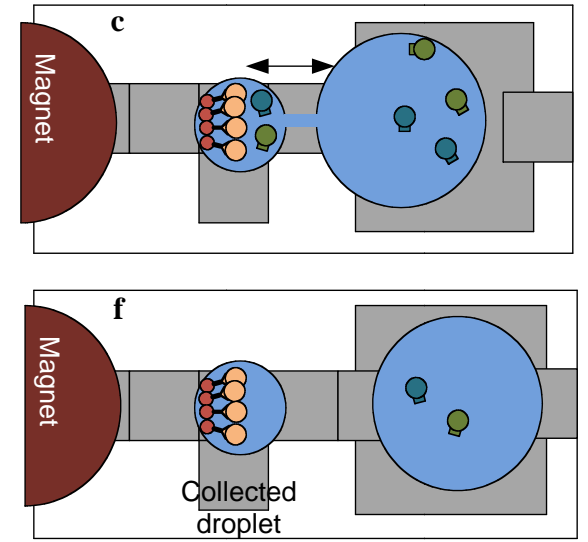

Figure 6: Schematic description of magnetic concentration of CD8+ cells. (a) Initial droplet containing fluorescently stained CD8+ cells (orange) preconjugated with MBs (red), and unlabeled CD8-cells (blue, green). (b) On introducing magnet, MB-bound CD8+ cells collected at left meniscus. $(c, d)$ Droplet is cut into collected droplet, containing the MB-CD8+cells, and depleted droplet with CD8- cells. (d-f) Remaining CD8- cells in the collected droplet can be removed by diluting it with a buffer droplet and repeating the process.

(a, e) Initial Cell Sample
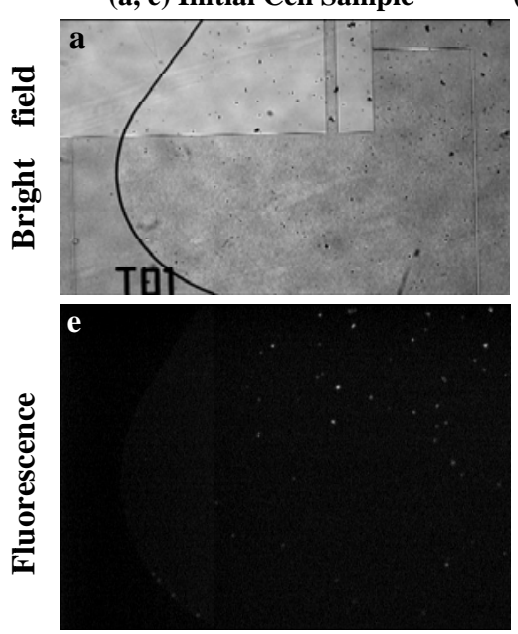

(b, f) MB-bound cells collected at left
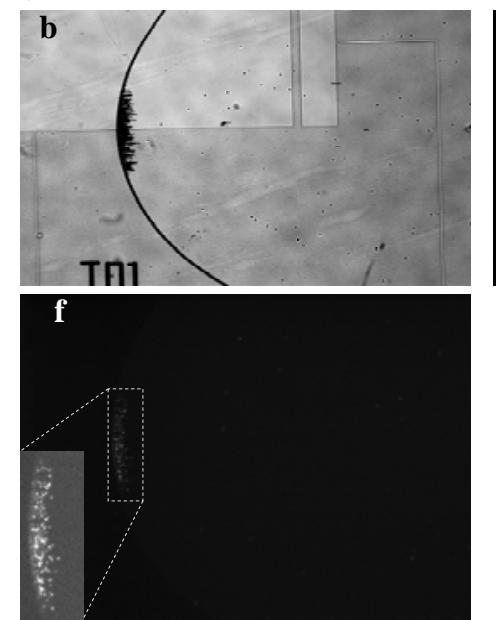
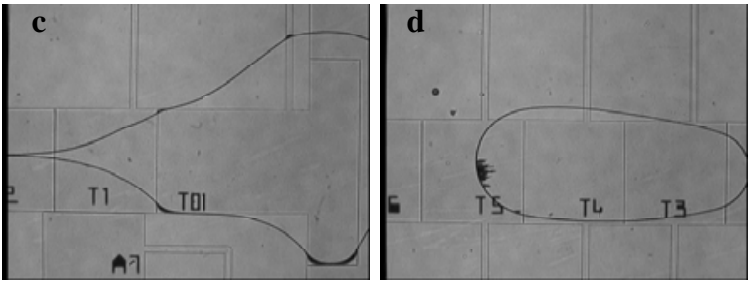

Figure 7: Concentration of CD8+ T-lymphocytes: (a,e) Initial cell sample with fluorescently stained CD8+ cells preconjugated with MB's, and unlabeled CD8- cells. $(b, f)$ Magnet is introduced from left (not in view), collecting the $M B$-bound fluorescent cells to the left meniscus ( $f$ inset). (c) Droplet pulled to left, and (d) split to separate the collected MB-bound cells from the CD8-depleted droplet.

\section{CONCLUSION}

We have demonstrated the binding of antibody-conjugated magnetic beads with CD8+ lymphocytes on an EWOD device. We also showed the separation of MB bound CD8+ lymphocytes from unbound CD8- lymphocytes, as a technique to concentrate the former. Improvement in concentration efficiency can be achieved through serial dilution, using EWOD microfluidic operations. The concentrated CD8+ cells can then be lysed, and the proteins from the lysate analyzed for monitoring of organ transplant patients, as per protocol.

Future work will be towards demonstrating the specificity of the MB-CD8+ cell binding on EWOD, and using less preprocessed blood derivatives as samples, so as to move closer to the laboratory protocol used to monitor organ transplant rejection. Cell-lysis and protein detection will also need to be integrated for the complete diagnostic device.

\section{ACKNOWLEDGEMENT}

This work is supported by NASA through Institute for Cell Mimetic for Space Exploration (CMISE), NIH through Pacific Southwest RCE (grant AI065359), and Intramural Seed Grant of the UCLA Department of Urology.

\section{REFERENCES}

[1] A K Cashion, O M Sabek, C J Driscoll, L W Gaber, A O Gaber, "Serial Peripheral Blood Cytotoxic Lymphocyte Gene Expression Measurements for Prediction of Pancreas Transplant Rejection," Transplantation Proceedings, 2006, vol. 38, pp. 3676-3677.

[2] J L Veale et al., "Noninvasive Diagnosis of Cellular and Antibody-Mediated Rejection by Perforin and Granzyme B in Renal Allografts," Human Immunology, 2006, vol. 67, pp. 777-786.

[3] G J Shah, E Pierstorff, D Ho, C-J Kim, "Meniscus-Assisted Magnetic Bead Trapping on Ewod-Based Digital Microfluidics for Specific Protein Localization," Proc. Transducers, Lyon, France, June 2007, pp. 707-710.

[4] Y Z Wang, Y Zhao, S K Cho, "In-Droplet Magnetic Beads Concentration and Separation for Digital Microfluidics," Proc. Transducers, Lyon, France, June 2007, pp. 711-714.

[5] Y Z Wang, Y Zhao, S K Cho, "Efficient in-Droplet Separation of Magnetic Particles for Digital Microfluidics," J. Micromechanics and Microengineering, 2007, vol. 17, pp. 2148-2156. 